\title{
An interactive videogame designed to optimize respiratory navigator efficiency in children undergoing cardiac magnetic resonance
}

Sean M Hamlet ${ }^{1,2^{*}}$, Jonathan D Suever ${ }^{2,3}$, Jonathan D Grabau ${ }^{2}$, Gregory J Wehner ${ }^{2,4}$, Moriel Vandsburger ${ }^{5,4}$, Kristin N Andres ${ }^{2}$, David Powell ${ }^{4}$, Vincent L Sorrell ${ }^{6,7}$, Brandon K Fornwalt ${ }^{5,3}$

From 19th Annual SCMR Scientific Sessions

Los Angeles, CA, USA. 27-30 January 2016

\section{Background}

Advanced cardiac magnetic resonance (CMR) acquisitions often require long scan durations that necessitate respiratory navigator gating. This is particularly important in children with limited ability to hold their breath. We hypothesized that visual feedback of diaphragm position using an interactive videogame during CMR would increase navigator efficiency and improve image quality in children.

\section{Methods}

A feedback videogame was developed using MATLAB. The navigator image provided within the Siemens Syngo user-interface (Figure 1A) was processed in real-time to

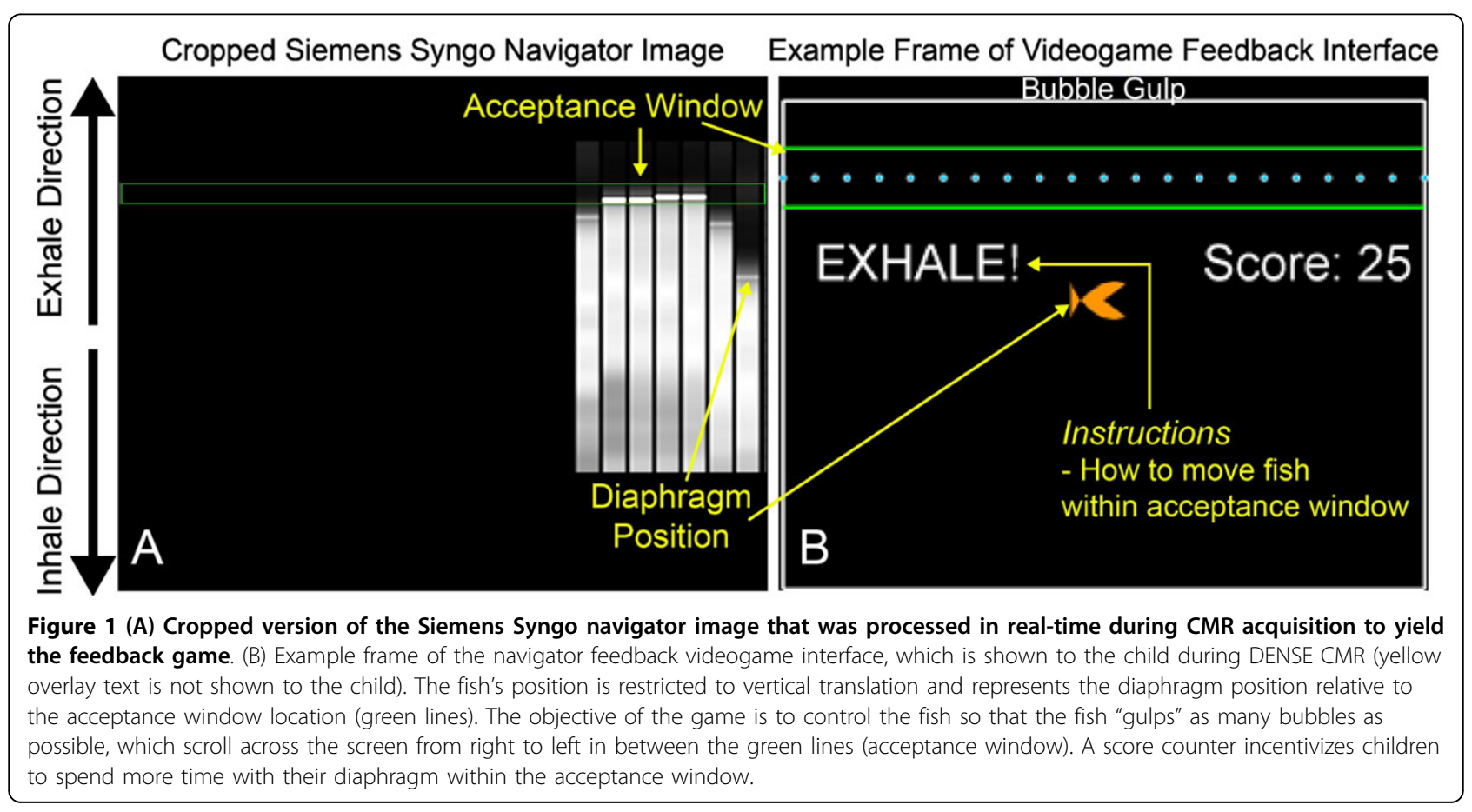

${ }^{1}$ Electrical and Computer Engineering, University of Kentucky, Lexington, KY, USA 

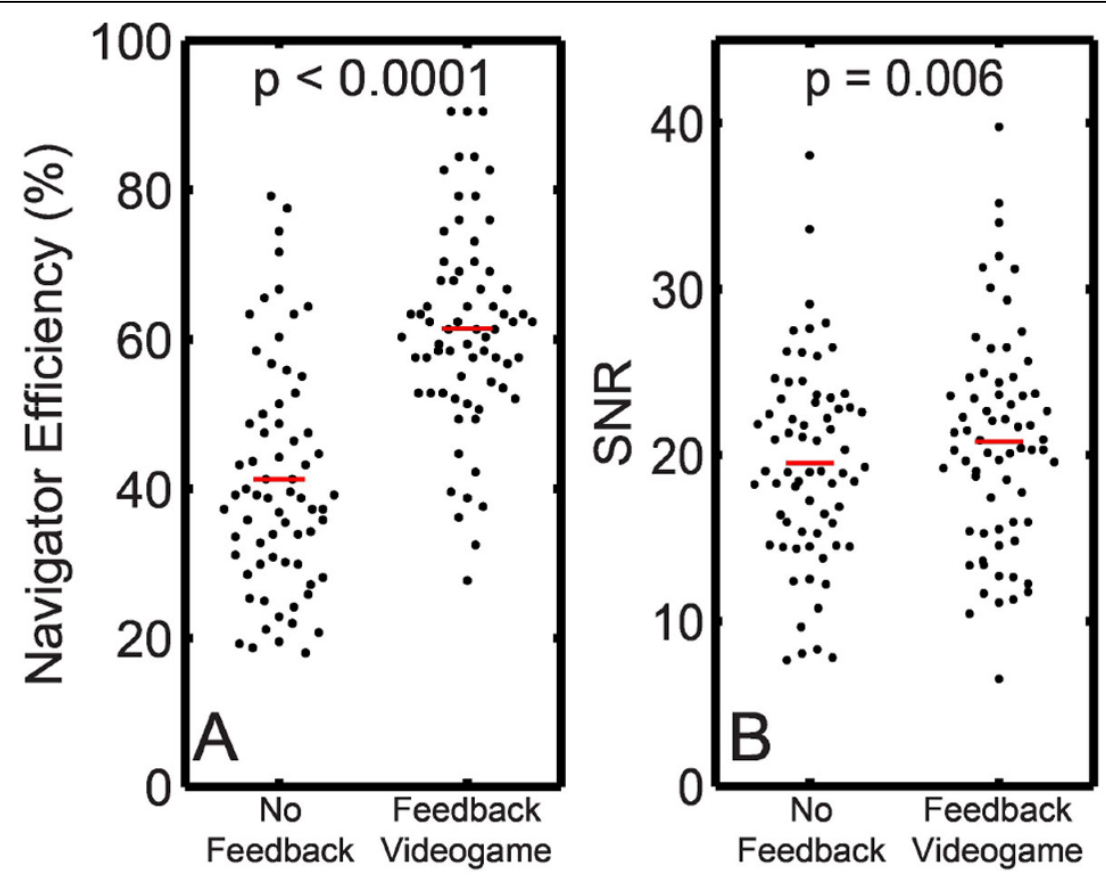

Figure 2 Use of a navigator videogame feedback system improves both navigator efficiency (A) and SNR (B) of DENSE images acquired in children.

yield a kid-friendly representation of diaphragm position which was then projected to the subject in the scanner (Figure 1B). The game used a point-based system to incentivize children to hold their diaphragm within the navigator acceptance window $( \pm 3 \mathrm{~mm})$ throughout image acquisition (Figure 1).

Using a 3T Siemens Tim Trio, 20 healthy children (Age: $13 \pm 3,35 \%$ female) underwent a navigator-gated 2D spiral cine displacement encoding with stimulated echoes (DENSE) acquisition (mid-ventricular, basal, apical, and 4-chamber images) first with no feedback and then with the videogame. Additional imaging parameters were: 12 spiral interleaves, voxel size: $2 \times 2 \times 8 \mathrm{~mm}$, TE/TR: $1.08 / 17$, flip angle: $20^{\circ}, 1$ average. Between the acquisitions with no feedback and those with the videogame, each child underwent two 30-heartbeat practice scans to familiarize themselves with the videogame. Navigator efficiency and signal-to-noise ratio (SNR) were determined for each subject and compared using a paired student's t-test.

\section{Results}

The videogame improved navigator efficiency by $50 \%$ ( $\mathrm{p}<$ 0.0001 , Figure $2 \mathrm{~A}$ ) and improved SNR by $7 \%$ compared to scans without feedback ( $p=0.006$, Figure $2 B$ ).

\section{Conclusions}

Use of a diaphragmatic feedback videogame during navigator-gated DENSE CMR can improve navigator efficiency by $50 \%$ in children. The videogame also has a slight positive effect on image quality with a $7 \%$ increase in SNR, potentially due to the shorter scan durations leading to reduced heart rate variability. These findings should be generalizable to all CMR acquisition sequences which utilize a respiratory navigator.

\section{Authors' details}

${ }^{1}$ Electrical and Computer Engineering, University of Kentucky, Lexington, KY, USA. ${ }^{2}$ Pediatrics, University of Kentucky, Lexington, KY, USA. ${ }^{3}$ Institute for Advanced Application, Geisinger Health System, Danville, PA, USA. ${ }^{4}$ Biomedical Engineering, University of Kentucky, Lexington, KY, USA. ${ }^{5}$ Physiology, University of Kentucky, Lexington, KY, USA. ${ }^{6}$ Medicine, University of Kentucky, Lexington, KY, USA. ${ }^{7}$ Cardiology, University of Kentucky, Lexington, KY, USA.

Published: 27 January 2016

doi:10.1186/1532-429X-18-S1-010

Cite this article as: Hamlet et al: An interactive videogame designed to optimize respiratory navigator efficiency in children undergoing cardiac magnetic resonance. Journal of Cardiovascular Magnetic Resonance 2016 18(Suppl 1):010. 